Published in final edited form as:

J Acquir Immune Defic Syndr. 2017 August 01; 75(4): e90-e98. doi:10.1097/QAI.0000000000001352.

\title{
30-day readmission rates in an HIV-infected cohort from Rio de Janeiro, Brazil
}

\author{
Lara E. Coelho, MD§, Sayonara R. Ribeiro, PhD, Andre M. Japiassu, PhD, Ronaldo I. \\ Moreira, MSc, Priscila C. Lara, MD, Valdilea G. Veloso, PhD, Beatriz Grinsztejn, and Paula \\ M. Luz, PhD \\ Instituto Nacional de Infectologia Evandro Chagas, FIOCRUZ, Rio de Janeiro, Brazil
}

\begin{abstract}
Background-The 30-day readmission rate is an indicator of the quality of hospital care and transition to the outpatient setting. Recent studies suggest HIV infection might increase the risk of readmission though estimates of 30-day readmission rates are unavailable among HIV infected individuals living in middle/low income settings. Additionally, factors that may increase readmission risk in HIV infected populations are poorly understood.
\end{abstract}

Methods-30-day readmission rates were estimated for HIV-infected adults from the Instituto Nacional de Infectologia Evandro Chagas/Fiocruz cohort in Rio de Janeiro, Brazil, from January 2007 to December 2013. Cox regression models were used to evaluate factors associated with the risk of 30-day readmission.

Results-Between January 2007 and December 2013, 3991 patients were followed and 1861 hospitalizations were observed. The estimated 30-day readmission rate was 14\% (95\% confidence interval $12.3 \%-15.9 \%$ ). Attending a medical visit within 30 days after discharge (aHR 0.73, $\mathrm{p}=0.048$ ) and being hospitalized in more recent calendar years (aHR $0.89, \mathrm{p}=0.002)$ reduced the risk of 30-day readmission. In contrast, low CD4 counts $\left(51-200\right.$ cells $/ \mathrm{mm}^{3}$ : aHR 1.70, p=0.024 and $\leq 50$ cells $/ \mathrm{mm}^{3}$ : aHR 2.05, $\mathrm{p}=0.003$ ), time since HIV infection diagnosis $\geq 10$ years (aHR 1.58, $\mathrm{p}=0.058$ ) and leaving hospital against medical advice (aHR 2.67, $\mathrm{p}=0.004)$ increased the risk of 30-day readmission.

Conclusions-Patients with advanced HIV/AIDS are most at risk of readmission and should be targeted with prevention strategies to reduce this risk. Efforts to reduce discharge against medical advice and to promote early post-discharge medical visit would likely reduce 30-day readmission rates in our population.

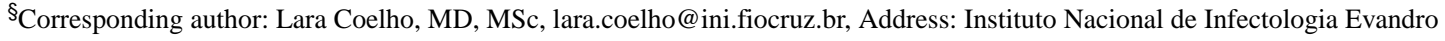
Chagas (INI), FIOCRUZ, Av Brasil 4365, Manguinhos, Rio de Janeiro, RJ, Brazil. Zip code: 21045-900.

Authors' contributions

LEC and PML conceived the study, performed the analyses, and drafted the manuscript. LEC, SRR and PCL reviewed hospitalizations' discharge summaries and followed the protocol for assigning ICD-10 codes/causes to all hospitalizations. AMJ and RIM were in charge of obtaining and reviewing patient data and were responsible for data harmonization. BG and VGV contributed to the study's design and were involved in revising the manuscript for important intellectual content. All authors read and approved the final manuscript.

Competing interests

The authors declare no competing interests.
} 


\section{Keywords}

HIV; hospitalization; readmission; healthcare utilization; length of stay; antiretroviral therapy

\section{Introduction}

Hospital readmission within 30 days of discharge, i.e. 30-day readmission, has been increasingly proposed as an indicator of the quality of hospital care and of transition care to the outpatient setting ${ }^{1}$. In Brazil, since 2015 the National Agency for Supplementary Health also incorporated 30-day readmission as an indicator of health care assistance quality ${ }^{2}$.

Between hospitals comparisons, adjusted for patients' characteristics and for the community within which a hospital is located ${ }^{3}$, can help identify institutions with worse performance allowing for adoption/reviewing of clinical protocols to improve hospital care ${ }^{4}$.

Economically, readmissions are expensive and consume a great share of health designated expenditures ${ }^{5}$. Altogether, studies have suggested that the keeping of 30-day readmission rates within the expected average values can result in a win-win scenario: improvement in hospital quality and efficiency allowing for better allocation of health expenditures.

In the United States, all-cause 30-day readmission rates in the general population is estimated at $14 \%{ }^{6}$. Additionally, of the overall 30 -day readmissions, $30 \%$ are thought to be potentially preventable events ${ }^{7}$. Several chronic medical conditions have been associated with high 30-day readmission rates with HIV being recently included among these conditions $^{8-10}$. A recent study conducted in the United States showed a 1.5-fold increase in the risk of readmission for HIV infected patients compared to their non-HIV infected counterparts ${ }^{10}$. Moreover, among HIV infected patients, the proportion of potentially preventable readmissions is suggested to be even higher, reaching $53 \%{ }^{11}$.

The study of 30-day readmission rates and its predictors among HIV infected patients can provide relevant information to guide monitoring of hospital quality and transition of care with the goal of reducing morbidity of the HIV infected population. To the best of our knowledge, studies addressing 30-day readmission rates and its determinants in HIV infected populations are restricted to high-income settings ${ }^{10-17}$ and the findings likely do not reflect low- to middle-income settings due to dissimilarities in infectious diseases burden as well as differences in the provision and structure of health care. In this study, we sought to 1) estimate 30-day readmissions rates among HIV patients living in a middle-income setting and 2) identify patient- and health care-level factors that were associated with higher risk of 30-day readmission among HIV infected patients from a cohort in Rio de Janeiro, Brazil, during the period of 2007-2013.

\section{Methods}

\section{Study site and population}

Since 1986, Instituto Nacional de Infectologia Evandro Chagas of Fundacao Oswaldo Cruz (INI/FIOCRUZ) has been a national referral center for infectious diseases care, research and training, being one of the largest providers of primary, specialty, and tertiary care for HIV 
infected individuals in Rio de Janeiro State. INI comprises an Infectious Disease tertiary hospital, Evandro Chagas Hospital, a day clinic (for chemotherapy and other parenteral drugs administration), an outpatient clinic and an emergency department, all funded by the Brazilian national health system (known as Sistema Único de Saúde). All HIV infected patients followed in the INI cohort have free-of-charge access to INI's facilities as well as to antiretroviral treatment and other medications (such as opportunistic infections prophylaxis, Kaposi sarcoma chemotherapy, and diabetes, hypertension medications, etc.) provided by the Brazilian national health system. A longitudinal, periodically updated, observational clinical database has been maintained on patients receiving HIV care at INI. The database includes socio-demographic, laboratory, clinical, and therapeutic information abstracted from the medical records by trained staff. Additionally, dates of admission and discharge to Evandro Chagas Hospital are also included in the database along with discharge diagnoses and summary. Cohort procedures have been described and results published ${ }^{18-20}$.

For the present study, the study population included adult ( $\geq 18$ years of age at cohort enrollment) HIV infected patients, enrolled in the INI cohort between 01 January 1985 and 01 December 2013, who were alive after 01 January 2007 and in active care (at least one medical visit after 01 January 2007) from 1997 to 2013.

\section{Outcome definition}

Index hospitalizations were defined as: 1) the first hospitalization in the study period or any hospitalization occurring >30 days after the most recent previous hospitalization, and 2) for which there was a live discharge. Hospitalizations occurring from 01 January 2007 (or from date of cohort enrollment for those enrolled after this date) until 31 December 2013 were considered. Patients were not censored after a hospitalization such that all hospitalization events during the years 2007-2013 were included. The only exceptions were the exclusion of hospitalizations (1) with admission date prior to 01 February 2007 because of the need to observe 30 days before a potential index hospitalization, and (2) with discharge date after 30 November 2013 because of the need to observed 30 days after a discharge for potential 30day readmissions. Hospital readmissions occurring within 30 days of an index hospitalization discharge were defined as 30-day readmissions. When a "chain" of multiple readmissions with less than 30-day intervals each followed an index hospitalization, all readmissions in the chain were excluded from being an index hospitalization. 30-day readmission rate was calculated by dividing the number of 30-day readmissions by the number of index hospitalizations.

\section{Independent variables}

Socio-demographic and clinical variables were evaluated for association with 30-day readmission risk. CD4 count and HIV viral load closest to index hospitalization were selected on a timeframe of 180 days before and 30 days after the hospital admission date. Antiretroviral therapy (ART) use prior to index hospitalization was defined as a dichotomous yes/no variable based on ART initiation any time before index hospitalization admission. Since we do not have information on adherence, after ART first prescription, participants were defined as on ART. Time since HIV infection diagnosis was calculated as the difference between date of index hospitalization admission and date of the first positive HIV 
test. Type of hospitalization discharge was based on discharge reports and categorized as follows: according to medical advice, against medical advice, transfer to another hospital facility and unknown. Length of stay (LOS) was calculated by subtracting hospital admission date from date of discharge and adding 1. Use of intensive care unit (ICU) (yes/no) was used as a proxy for the severity of the patient's clinical condition. Early transition from hospital to outpatient care was evaluated through the presence of a medical visit within 30-days of index hospitalization discharge or before readmission for those readmitted within 30-days.

Hospitalization discharge reports were reviewed and all the diagnoses listed in the discharge report were classified using the $10^{\text {th }}$ Edition of the International Classification of Disease (ICD-10), into 24 different categories ${ }^{19}$. Since some ICD-10 codes could be allocated to several categories, we considered a hierarchical classification scheme with a decreasing order of priority as follows: AIDS-events, non-AIDS malignancies, infections and then systemic events ${ }^{19}$. Subsequently, to determine the primary cause of a hospitalization, the one or more ICD-10 codes listed in the discharge reports were hierarchically classified as follows: AIDS-defining diseases, non-AIDS malignancies, cardiovascular disease, bacterial infections, fungal infections, viral infections, parasitic infection, digestive diseases, renal diseases, respiratory diseases, neurologic diseases, endocrine diseases, hematological diseases, psychiatric diseases, viral hepatitis, non-viral hepatitis, dermatological diseases, rheumatologic diseases, trauma, gynecologic disease, toxicities, others and sings and symptoms.

\section{Statistical analysis}

The evaluation of the factors associated with 30-day readmission risk was performed using Cox extended regression models (accounting for clusters within patients). Patient's followup started on the day of discharge from the index hospitalization and ended at the readmission date, date of death or 30 days after index discharge, whichever occurred first. Variables with p-value below 0.10 in the unadjusted analysis were included in the adjusted model in order to explore their effects after adjusting for all potential confounding. The variable "medical visit within 30-day of discharge" was included in the model as a timedependent variable such that a patient's follow up time was split into before and after the medical visit for those who attended a medical visit within 30-days of discharge. Using this approach, we also prevented a potential time-dependent bias, whereas patients who are soon readmitted have a decreased chance of having a medical visit after discharge. Proportional hazards assumption was tested using Schoenfeld residuals. R (version 3.0.3) and library "survival" were used for the analyses.

\section{Ethical considerations}

This study was approved by the ethics committee of the INI (CAAE 0032.0.009.000-10) and was conducted according to the principles expressed in the Declaration of Helsinki. All patient records/information were de-identified prior to analysis. 


\section{Results}

During the study period, from the 3991 included patients, 3014 were never hospitalized while 977 had at least one hospitalization during the study period. In total, 1861 hospitalization events occurred: 1442 were considered index hospitalizations, 202 were 30day readmissions, and 16 index hospitalizations were followed by death after discharge (without readmission) (Figure 1). Thirty-day readmission rate was estimated as 14.0\% (95\% confidence interval [CI] 12.3\%-15.9\%). For those who readmitted, the median time between index discharge and readmission was 14 days (interquartile range [IQR]: 7-21). The three most frequent causes of index hospitalizations were AIDS-defining diseases (688/1442, $47.7 \%)$, bacterial infections $(289 / 1442,20.0 \%)$, and cardiovascular diseases $(117 / 1442$, 8.1\%) (Figure 2). 30-day readmission rate among AIDS-defining diseases index hospitalizations reached $18.8 \%$ (95\% CI 15.9\%-21.9\%) which is higher than the overall rate. The highest readmission rate was observed for endocrine diseases $(20.0 \%, 95 \% \mathrm{CI}$ $3.5 \%-55.8 \%)$ which comprised only $0.7 \%(\mathrm{n}=10)$ of the index hospitalizations (Figure 2$)$. Among the 202 readmissions, the most frequent cause of hospitalization was AIDS-defining diseases $(129 / 202,63.9 \%)$ and the second most frequent cause was bacterial infections $(36 / 202,17.8 \%)$.

Patients' characteristics at the moment of the index hospitalization admission (stratified by yes/no 30-day readmission), as well as the unadjusted hazard ratios (cHR) are shown in Table 1. Compared to patients not readmitted, those who were readmitted were more likely to have recent ( 30 days) HIV infection diagnosis ( $14.9 \%$ vs. $8.5 \%$ among non-readmitted, Chi-squared $\mathrm{p}$-value $=0.019)$ and lower CD4 counts (median 118 cells $/ \mathrm{mm}^{3}$ vs. 191 cells $/ \mathrm{mm}^{3}$ among non-readmitted, Wilcoxon Rank-Sum test $\mathrm{p}$-value $\left.<0.0001\right)$. On the other hand, patients who were readmitted were less likely to have used ART prior to index hospitalization (66.3\% vs. $76.3 \%$ among non-readmitted, Chi-squared p-value $=0.003)$ and to attend a medical visit within 30 days after index discharge (37.6\% vs. $74.7 \%$ among nonreadmitted, Chi-squared p-value $<0.001)$. Moreover, index hospitalizations that were followed by 30-day readmissions had longer LOS (median 16 days vs. 12 days among nonreadmitted, Wilcoxon Rank-Sum test p-value $<0.001$ ), had higher frequency of ICU stay ( $15.3 \%$ vs. $9.4 \%$ among non-readmitted, Chi-squared p-value $=0.015)$, and more likely ended with a discharge against medical advice (5.4\% vs. $2.5 \%$ among non-readmitted, Chi-squared p-value $=0.029$ ).

In unadjusted models, recent HIV infection diagnosis ( $\$ 30$ days), CD4 counts bellow 200 cells $/ \mathrm{mm}^{3}$, LOS longer than 21 days, ICU stay and discharge against medical advice significantly increased the risk of 30-day readmission. On the other hand, ART use prior to index hospitalization, being hospitalized in most recent calendar years and attending medical visit within 30 days after discharge protected against 30-day readmission (though this last variable had a p-value $=0.072)($ Table 1$)$.

The final adjusted model is shown in Figure 3. Comparing the effects of the independent variables in the unadjusted and adjusted models, we found that after adjusting for covariates, the protective effect of attending a medical visit within 30 days of discharge remained similar but it gained statistical significance (adjusted hazard ratio [aHR] 0.73, p- 
value $=0.048$ ). Being hospitalized in most recent years also remained significant in the adjusted models (aHR 0.89, $\mathrm{p}$-value $=0.002$ ). The protective effect of ART use prior to hospitalization observed in the unadjusted model was attenuated but the point estimate remained protective (although not statistically significant) after controlling for covariates $($ aHR 0.73, p-value $=0.113)$. On the other hand, CD4 counts $\leq 50$ cells $/ \mathrm{mm}^{3}($ aHR 2.05, pvalue $=0.003)$ and between 200-51 cells $/ \mathrm{mm}^{3}($ aHR 1.70, $\mathrm{p}$-value $=0.024)$ and leaving the hospital against medical advice $(\mathrm{aHR} 2.67$, p-value $=0.004)$ increased the risk of 30-day readmission. Additionally, after adjusting for covariates, having HIV infection diagnosis for 10 or more years also borderline increased the risk of 30-day readmission (aHR 1.58, pvalue $=0.058$ )

\section{Discussion}

In the present study, we have estimated the 30-day readmission rate for an HIV infected cohort followed in Rio de Janeiro, Brazil. Our estimated 14\% readmission rate is lower than the rates reported for HIV infected patients in high-income settings. Nijhawan et al. estimated a $25 \%$ readmission rate in Dallas, US ${ }^{17}$, Berry at al. reported $19 \%$ readmission rate for the HIV Research Network (HIVRN, which includes 12 sites in the US) ${ }^{16}$, and a US national report (using data from Healthcare Cost and Utilization Project) found a readmission rate of $20 \%$ for HIV infected patients ${ }^{10}$. The reasons for the disparate readmission rates likely include patient, hospital and health care system as well as community characteristics ${ }^{3}$ beyond those that we have been able to address. Nonetheless, patient-level characteristics shown to increase the risk of readmission, such as those indicative of advanced disease, were similar among ours and the previous studies ${ }^{16,17,21}$, highlighting sub-populations for whom efforts could be targeted. Moreover, our results shed light on the benefits of an early post-discharge medical visit in preventing readmission.

After adjusting for potential confounding variables, having an early post discharge medical visit reduced the risk of readmission by $27 \%$ in our study population. This finding is in agreement with a previously described protective effect of careful transitioning to the outpatient setting on reducing the risk of readmission among patients with other chronic conditions ${ }^{22-25}$. Additionally, it might indicate a better post discharge support system (i.e family and social support) that has also been associated with lower risk of hospital readmission ${ }^{24,26}$. Interestingly, among HIV-infected patients, only one study reported on this association and found no link between early transition to outpatient care and 30-day readmission ${ }^{16}$. Thus, although our results are consistent with previous studies that included patients with other chronic diseases, confirmatory studies are needed to provide more reliable information on the effect of attending an outpatient visit on 30-day readmission rates for the HIV infected population.

Our study corroborates results reported by Berry et al, Feller et al and Nijhawan et al ${ }^{16,17,21}$ by showing that a proxy of advanced HIV/AIDS disease such as low CD4 counts at index hospitalization is associated with an increased risk of readmission. This finding highlights the impact that advanced chronic diseases ${ }^{27,28}$, in our case, advanced HIV disease, has on 30-day readmission risk. To focus clinical efforts in this severely ill sub-population seems feasible given they represent only a small fraction of patients under follow-up. For the 
present cohort, this sub-population would amount to approximately $15 \%$ of the patients under follow-up as only 588 patients had an AIDS-defining disease or poor immune function at index hospitalization out of 3991 patients under follow-up. Additionally, ART use prior to the index hospitalization may likely reduced the risk of 30-day readmission (though this result was not statistically significant in the present analysis). Importantly, when we stratified ART use into recent start ( $\leq 30$ days) or not (more than 30 days of use prior to index hospitalization admission) we did not find an increased risk of readmission associated with recent ART start that could be linked to Immune Reconstitution Inflammatory Syndrome and/or acute toxic events (data not shown). Similarly, both Berry et al and Nijhawan et al explored this possible risk association (recent ART and readmission risk) and neither found an association ${ }^{16,17}$. Taken together, these findings suggest that early ART use for all HIV-infected patients might not only prevent HIV disease progression and HIV transmission but may as well reduce 30 -day readmission.

Another interesting finding from our study was the fact that long term HIV infected patients, i.e. those diagnosed for 10 or more years, were at higher risk of 30-day readmissions after controlling for all other covariates (including age). We can speculate on some plausible explanations for this finding. It is possible that some chronically HIV infected patients, likely heavily ART experienced, are presenting with multidrug HIV resistance and/or poor adherence and therefore disease progression, leading to increased risk of readmission. This hypothesis is supported by the fact that although $94.2 \%$ of those patients had used ART prior to index hospitalization, less than half $(47 \%)$ had a viral load $<400$ copies $/ \mathrm{mL}$ (data not shown). Another possible reason would be the fact that HIV chronic infection leads to persistent inflammation and immune activation which is associated with increased risk of cardiovascular disease, cancer and other non-AIDS related events ${ }^{29,30}$. Corroborating this hypothesis, we found that index hospitalizations among patients with HIV diagnosis for 10 or more years were less likely to be due to an AIDS defining disease (29\% vs. $47 \%$ among all index hospitalizations), and that the readmission rate for digestive diseases index hospitalizations surpassed the one for AIDS-defining diseases index hospitalizations (23.8\% vs. $21.4 \%$ ) highlighting the importance of non-AIDS events on the risk of readmission for this particular population subset. In summary, a multicausal pathway that encompasses aging, higher prevalence of non-communicable diseases, ART failure seems to be a more plausible explanation for the increased readmission risk observed among HIV long term infected patients.

Additionally, our results show that patients who left the hospital against medical advice had a two-fold higher risk of 30-day readmission. Indeed, discharge against medical advice had already been associated with increased risk of 30-day readmission in the general population $^{31}$ and among those with HIV ${ }^{13,21,32}$. Interestingly, compared to HIV-uninfected patients, HIV infected patients were shown to have a higher risk of leaving the hospital against medical advice ${ }^{32}$. Specifically, among HIV infected patients, leaving hospital against medical advice led to a 5-fold increased odds of 30-day readmission for a related diagnosis and was associated with longer readmission LOS (compared to those who had been formally discharged $)^{13}$. Altogether, interventions focusing on reducing such discharges are needed to improve patients' health and reduce unnecessary costs with readmissions. 
AIDS-defining diseases were the main cause of index hospitalizations comprising almost half of all admissions, followed by bacterial infections. Endocrine diseases had the highest readmission rate in our study population, a finding that results from the very small number of index hospitalizations (i.e. small denominator) while AIDS-defining diseases led to the second highest readmission rate. These findings are somewhat different from those observed in previous studies. In the HIVRN population, non-AIDS infections were the main cause of index hospitalizations, and the highest 30-day readmission rate was observed for oncologic index hospitalizations, with the second highest 30-day readmission rate for an AIDSdefining disease index hospitalization ${ }^{16}$. Nijhawan et al also found AIDS-defining diseases as the main cause of index hospitalizations but the oncologic hospitalizations led the 30-day readmission rates and AIDS-related diseases came in fifth ${ }^{17}$. Several factors can explain these differences in the rating of the causes of index hospitalizations and readmissions. Foremost, the burden of AIDS diseases in the context of hospitalizations is quite different between our study population and HIVRN population. In our population, the proportion of index hospitalizations due to AIDS-defining diseases was 5-fold higher than that observed for the HIVRN population (47.7\% vs. $9.6 \%)^{16}$ likely driving the ranking for both index hospitalizations and readmissions. Additionally, differences in the burden of cancer among our study population and HIV-infected populations from high-income settings might explain some of these disparities. A recent study compared the incidence of cancer (AIDS and nonAIDS defining) between HIV infected patients from INI cohort and Vanderbilt Comprehensive Care Clinic cohort (Nashville, US) and showed significant differences on the incidence of cancer between these two HIV-infected populations ${ }^{33}$. They showed that the incidence of AIDS defining cancer was higher in INI than in Vanderbilt, mainly driven by Kaposi sarcoma incidence (almost three times higher in INI). On the other hand, non-AIDS defining cancer incidence was higher in Vanderbilt than in INI, and the most incident cancer types were also different (anal cancer in INI vs. lung cancer in Vanderbilt). It is worthwhile mentioning that although INI provides treatment of Kaposi sarcoma, mostly administrated in a day clinic (including monitoring and management of potential side effects such as leukopenia), all other oncologic and chemotherapy-relate hospitalization are referred to another facility and thus not included in this analysis. As such, the findings regarding oncologic admissions and readmissions should be compared with caution to other published results.

Overall, the median LOS of index hospitalizations (12 days) surpasses the one reported for the HIVRN population (median of 5 days) ${ }^{16}$ and the one reported by Nijhawan et al (mean 7 days) ${ }^{17}$ but is closer to a national HIV-infected Portuguese estimate (median 11 days) ${ }^{34}$. And similarly to Berry et $\mathrm{al}^{16}$, we found that compared with other causes, hospitalizations due to AIDS defining diseases were associated with longer LOS (median of 18 vs. 9 days for AIDS and non-AIDS related hospitalizations, respectively, data not shown). Differences in hospital structure, hospital setting and health system level factors could be the sources of such disparities. For the purpose of comparison with Brazilian data, we searched publicly available databases that provide mean LOS and found that overall the mean LOS estimated for the state of Rio de Janeiro in the period of 2008-2015 was 9 days, while, for the same period, the national mean was 7 days revealing the high heterogeneity that exists when it comes to hospital outcomes even within Brazil ${ }^{35}$. 
There are several limitations that need to be highlighted in the present study, some of which were already addressed throughout this discussion. First, the comparison of readmission rates in our setting with that reported in the United States and other high-income countries must be made cautiously since there are hospital and health systems level factors, in addition to patient level factors, that can influence this outcome. In fact, such factors likely influence two other outcomes related to hospitalizations, LOS and in-hospital mortality, for which published results can be compared. The median index hospitalization LOS observed in our study is $>2$-fold higher than previously described in a US study (12 days versus 5 days reported by Berry et $\mathrm{al}^{16}$ ). One explanation for this difference is the primary cause of the hospitalization as AIDS-related hospitalizations are associated with longer LOS than nonAIDS related ones both in high- and middle-income settings ${ }^{36-38}$. Moreover, in our study, AIDS-related hospitalizations comprised almost half of the hospitalizations while in Berry et al. in represented only $9.6 \%$ of the index hospitalizations ${ }^{16}$. In addition, the pressure to reduce LOS in US hospitals has led to significant reductions in $\operatorname{LOS}^{39}$ while this is much less present in the Brazilian public health system. An examination of in-hospital mortality results also shed light on the discussion. In a previous study from our hospital, we found that in-hospital mortality for HIV-infected patients was $9.2 \%$, which contrasts with the $2.6 \%$ mortality rate reported by a tertiary hospital in New York ${ }^{40}$. Of note, the potential inverse correlation between in-hospital mortality and readmission risk (i.e. the higher the mortality rate the lower the readmission ${ }^{41}$ ) may also be playing a role.

Second, our study sample is from a single cohort that has access to an outpatient clinic as well as an infectious diseases hospital located in Rio de Janeiro, hence our results may not reflect those for other HIV populations in Brazil. Third, hospitalizations at Evandro Chagas hospital are restricted to non-surgical and non-obstetrics procedures, and, as mentioned earlier, oncologic hospitalizations (except for Kaposi sarcoma) are mainly referred to an oncological hospital and therefore our rates do not represent the entire sort of events that can happen to an HIV infected patient. Furthermore, we cannot rule out the occurrence of readmissions to other hospitals, implying that our readmission rate may be underestimated. Finally, other factors, not addressed in the present study, were found associated with readmission and merit further investigation including socioeconomic (income, housing stability, social/family support), behavioral (alcohol use) as well as comorbidities (in particular mental illnesses). For instance, Feller et al., while studying readmission in HIV infected patients in New York State, identified that multiple comorbidities, alcohol abuse, diagnosis of depression or psychosis, previous hospitalizations and emergency department visits and unstable housing all increased the risk of 30-day readmission ${ }^{21}$. Additionally, recent studies have shown that functional status at index hospitalization can also be associated with early readmissions ${ }^{42,43}$.

In summary, we found that the 30-day readmission rate in our HIV infected cohort of patients was $14 \%$. To our knowledge, ours is the first study to estimate and address 30-day readmission among HIV infected individuals in Brazil and, as such, provides a benchmark until more comprehensive data is available for the country. We also showed that attending an early medical visit after discharge had an important effect on preventing readmissions; this finding could be used to create/modify transition protocols to reduce readmission rates in the future. Finally, we demonstrated that patients with advanced HIV/AIDS (i.e. low CD4 
counts) have higher risk of readmission and that ART may reduce this risk, thus indicating early HIV diagnosis and prompt ART start as strategies that could reduce 30-day readmissions.

\title{
Acknowledgments
}

\begin{abstract}
Acknowledgements and funding
PML and BG acknowledge funding from the National Council of Technological and Scientific Development (CNPq) and the Research Funding Agency of the State of Rio de Janeiro (FAPERJ). This work was supported in part by the NIH-funded Caribbean, Central and South America network for HIV epidemiology (CCASAnet), a member cohort of the International Epidemiologic Databases to Evaluate AIDS (leDEA) (U01AI069923).
\end{abstract}

\section{References}

1. Hansen LO, Young RS, Hinami K, Leung A, Williams MV. Interventions to reduce 30-day rehospitalization: a systematic review. Ann Intern Med. 2011; 155(8):520-528. [PubMed: 22007045]

2. Brasil. Agência Nacional de Saúde Suplementar - ANS. [Accessed February 2, 2017] Fichas Técnicas e Nota técnica n ${ }^{\circ}$ 34/DIDES. Indicadores do Fator de Qualidade. Dez. 2015 http:// www.ans.gov.br/images/stories/Plano_de_saude_e_Operadoras/Area_do_prestador/ contrato_entre_operadoras_e_prestadores/indicadores-e-nota.pdf

3. Joynt KE, Jha AK. Thirty-day readmissions--truth and consequences. N Engl J Med. 2012; 366(15): 1366-1369. DOI: 10.1056/NEJMp1201598 [PubMed: 22455752]

4. Dharmarajan K, Hsieh AF, Lin Z, et al. Hospital readmission performance and patterns of readmission: retrospective cohort study of Medicare admissions. BMJ. 2013; 347(nov19 23):f6571f6571. DOI: 10.1136/bmj.f6571 [PubMed: 24259033]

5. Jencks SF, Williams MV, Coleman EA. Rehospitalizations among patients in the Medicare fee-forservice program. N Engl J Med. 2009; 360(14):1418-1428. [PubMed: 19339721]

6. Barret ML, Wier LM, Jiang J, Steiner CA. All-Cause Readmissions by Payer and Age, 2009-2013. Agency Healthc Res Qual HCUP Stat Brief. 2015; 199

7. van Walraven C, Bennett C, Jennings A, Austin PC, Forster AJ. Proportion of hospital readmissions deemed avoidable: a systematic review. CMAJ Can Med Assoc J J Assoc Medicale Can. 2011; 183(7)doi: 10.1503/cmaj.101860.

8. Ross JS, Mulvey GK, Stauffer B, et al. Statistical models and patient predictors of readmission for heart failure: a systematic review. Arch Intern Med. 2008; 168(13):1371-1386. [PubMed: 18625917]

9. Mannino DM, Thomashow B. Reducing COPD readmissions: great promise but big problems. Chest. 2015; 147(5):1199-1201. DOI: 10.1378/chest.15-0380 [PubMed: 25940241]

10. Berry S, Fleishman J, Moore R, Gebo K. Thirty-day hospital readmissions for adults with and without HIV infection: HIV readmissions. HIV Med. Aug.2015 n/a - n/a. doi: 10.1111/hiv.12287.

11. Nijhawan AE, Kitchell E, Etherton SS, Duarte P, Halm EA, Jain MK. Half of 30-Day Hospital Readmissions Among HIV-Infected Patients Are Potentially Preventable. AIDS Patient Care STDs. 2015; 29(9):465-473. DOI: 10.1089/apc.2015.0096 [PubMed: 26154066]

12. Barba Martin R, Marco Martinez J, Plaza Canteli S, et al. Retrospective study of early readmissions at an internal medicine service. Rev Clin Esp. 2000; 200(5):252-256. [PubMed: 10901002]

13. Anis AH, Sun H, Guh DP, Palepu A, Schechter MT, O’Shaughnessy MV. Leaving hospital against medical advice among HIV-positive patients. Can Med Assoc J. 2002; 167(6):633-637. [PubMed: 12358196]

14. Chew KW, Yen IH, Li JZ, Winston LG. Predictors of Pneumonia Severity in HIV-Infected Adults Admitted to an Urban Public Hospital. AIDS Patient Care STDs. 2011; 25(5):273-277. DOI: 10.1089/apc.2010.0365 [PubMed: 21488749] 
15. Hsieh Y-H, Rothman RE, Bartlett JG, Yang S, Kelen GD. HIV seropositivity predicts longer duration of stay and rehospitalization among nonbacteremic febrile injection drug users with skin and soft tissue infections. JAIDS J Acquir Immune Defic Syndr. 2008; 49(4):398-405. [PubMed: 19186352]

16. Berry SA, Fleishman JA, Yehia BR, et al. Thirty-day hospital readmission rate among adults living with HIV. AIDS. 2013; 27(13):2059-2068. DOI: 10.1097/QAD.0b013e3283623d5f [PubMed: 23612008]

17. Nijhawan AE, Clark C, Kaplan R, Moore B, Halm EA, Amarasingham R. An electronic medical record-based model to predict 30-day risk of readmission and death among HIV-infected inpatients. JAIDS J Acquir Immune Defic Syndr. 2012; 61(3):349-358. [PubMed: 23095935]

18. Grinsztejn B, Veloso VG, Friedman RK, et al. Early mortality and cause of deaths in patients using HAART in Brazil and the United States. AIDS. 2009; 23(16):2107-2114. DOI: 10.1097/QAD. 0b013e32832ec494 [PubMed: 19770698]

19. Ribeiro SR, Luz PM, Campos DP, et al. Incidence and determinants of severe morbidity among HIV-infected patients from Rio de Janeiro, Brazil, 2000-2010. Antivir Ther. 2014; 19(4):387-397. DOI: 10.3851/IMP2716 [PubMed: 24445387]

20. Grinsztejn, B., Luz, PM., Pacheco, AG., et al. Changing Mortality Profile among HIV-Infected Patients in Rio de Janeiro, Brazil: Shifting from AIDS to Non-AIDS Related Conditions in the HAART Era. In: Yazdanpanah, Y., editor. PLoS ONE. Vol. 8. 2013. p. e59768

21. Feller DJ, Akiyama M, Gordon P, Agins BD. Readmissions in HIV-infected Inpatients: A Large Cohort Analysis. JAIDS J Acquir Immune Defic Syndr. Oct.2015 :1.doi: 10.1097/QAI. 0000000000000876.

22. Coleman EA, Parry C, Chalmers S, Min S. The care transitions intervention: results of a randomized controlled trial. Arch Intern Med. 2006; 166(17):1822-1828. [PubMed: 17000937]

23. Peikes D, Chen A, Schore J, Brown R. Effects of care coordination on hospitalization, quality of care, and health care expenditures among Medicare beneficiaries: 15 randomized trials. Jama. 2009; 301(6):603-618. [PubMed: 19211468]

24. Sharma G, Kuo Y-F, Freeman JL, Zhang DD, Goodwin JS. Outpatient follow-up visit and 30-day emergency department visit and readmission in patients hospitalized for chronic obstructive pulmonary disease. Arch Intern Med. 2010; 170(18):1664-1670. [PubMed: 20937926]

25. Chen H, Tisminetzky M, Lapane KL, et al. Decade-Long Trends in 30-Day Rehospitalization Rates After Acute Myocardial Infarction. J Am Heart Assoc. 2015; 4(11):e002291.doi: 10.1161/JAHA. 115.002291 [PubMed: 26534862]

26. Arbaje AI, Wolff JL, Yu Q, Powe NR, Anderson GF, Boult C. Postdischarge environmental and socioeconomic factors and the likelihood of early hospital readmission among communitydwelling Medicare beneficiaries. The Gerontologist. 2008; 48(4):495-504. [PubMed: 18728299]

27. Berry CE, Kalhan R. Chronic Obstructive Pulmonary Disease Rehospitalization. A Big Problem that Now Needs Solutions. Ann Am Thorac Soc. 2015; 12(12):1741-1742. DOI: 10.1513/ AnnalsATS.201510-687ED [PubMed: 26653184]

28. Desai AS, Stevenson LW. Rehospitalization for Heart Failure: Predict or Prevent? Circulation. 2012; 126(4):501-506. DOI: 10.1161/CIRCULATIONAHA.112.125435 [PubMed: 22825412]

29. Erlandson KM, Campbell TB. Inflammation in Chronic HIV Infection: What Can We Do? J Infect Dis. 2015; 212(3):339-342. DOI: 10.1093/infdis/jiv007 [PubMed: 25583171]

30. Deeks SG, Lewin SR, Havlir DV. The end of AIDS: HIV infection as a chronic disease. The Lancet. 2013; 382(9903):1525-1533. DOI: 10.1016/S0140-6736(13)61809-7

31. Glasgow JM, Vaughn-Sarrazin M, Kaboli PJ. Leaving Against Medical Advice (AMA): Risk of 30-Day Mortality and Hospital Readmission. J Gen Intern Med. 2010; 25(9):926-929. DOI: 10.1007/s11606-010-1371-4 [PubMed: 20425146]

32. Alfandre D, Yang J, Harwood K, et al. "Against Medical Advice” Discharges Among HIV-Infected Patients: Health and Health Services Outcomes. J Assoc Nurses AIDS Care. 2017; 28(1):95-104. DOI: 10.1016/j.jana.2016.10.002 [PubMed: 27815018]

33. Castilho JL, Luz PM, Shepherd BE, et al. HIV and cancer: a comparative retrospective study of Brazilian and U.S. clinical cohorts. Infect Agent Cancer. 2015; :10.doi: 10.1186/1750-9378-10-4. [PubMed: 25810759] 
34. Catumbela E, Freitas A, Lopes F, et al. HIV disease burden, cost, and length of stay in Portuguese hospitals from 2000 to 2010: a cross-sectional study. BMC Health Serv Res. 2015; 15(1)doi: 10.1186/s12913-015-0801-8.

35. Ministério da Saúde. Departamento de informática do SUS (DATASUS). Sistema de Informações Hospitalares do SUS (SIH/SUS). Brasil: www.datasus.gov.br [Access date: 02/02/2016]

36. Krentz HB, Dean S, Gill MJ. Longitudinal assessment (1995-2003) of hospitalizations of HIVinfected patients within a geographical population in Canada. HIV Med. 2006; 7(7):457-466. [PubMed: 16925732]

37. Berry SA, Fleishman JA, Moore RD, Gebo KA. Trends in reasons for hospitalization in a multisite United States cohort of persons living with HIV, 2001-2008. J Acquir Immune Defic Syndr 1999. 2012; 59(4):368-375. DOI: 10.1097/QAI.0b013e318246b862

38. Coelho LE, Ribeiro SR, Veloso VG, Grinsztejn B, Luz PM. Hospitalization rates, length of stay and in-hospital mortality in a cohort of HIV infected patients from Rio de Janeiro, Brazil. Braz J Infect Dis. Dec.2016 doi: 10.1016/j.bjid.2016.10.007.

39. Kaboli PJ, Go JT, Hockenberry J, et al. Associations between reduced hospital length of stay and 30-day readmission rate and mortality: 14-year experience in 129 Veterans Affairs hospitals. Ann Intern Med. 2012; 157(12):837-845. [PubMed: 23247937]

40. Kim JH, Psevdos G, Gonzalez E, Singh S, Kilayko MC, Sharp V. All-cause mortality in hospitalized HIV-infected patients at an acute tertiary care hospital with a comprehensive outpatient HIV care program in New York City in the era of highly active antiretroviral therapy (HAART). Infection. 2013; 41(2):545-551. DOI: 10.1007/s15010-012-0386-7 [PubMed: 23264096]

41. Gorodeski EZ, Starling RC, Blackstone EH. Are all readmissions bad readmissions? N Engl J Med. 2010; 363(3):297-298. [PubMed: 20647209]

42. Shih SL, Gerrard P, Goldstein R, et al. Functional Status Outperforms Comorbidities in Predicting Acute Care Readmissions in Medically Complex Patients. J Gen Intern Med. 2015; 30(11):16881695. DOI: 10.1007/s11606-015-3350-2 [PubMed: 25956826]

43. Greysen SR, Stijacic Cenzer I, Auerbach AD, Covinsky KE. Functional Impairment and Hospital Readmission in Medicare Seniors. JAMA Intern Med. 2015; 175(4):559.doi: 10.1001/ jamainternmed.2014.7756 [PubMed: 25642907] 


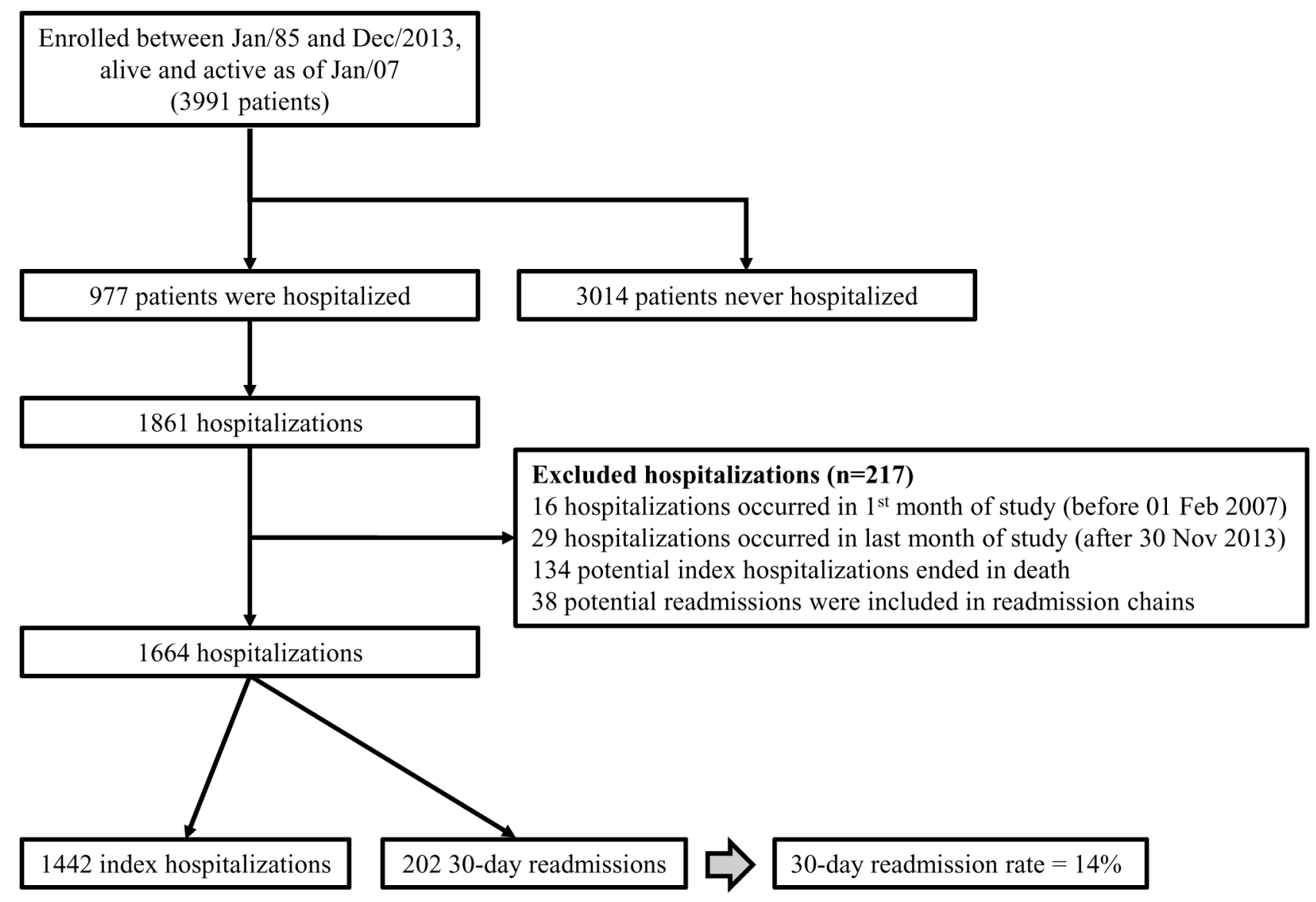

Figure 1. Patients and hospitalizations

Flowchart showing patient selection, total number of hospitalizations, hospitalization exclusion criteria and 30-day readmission rate. 

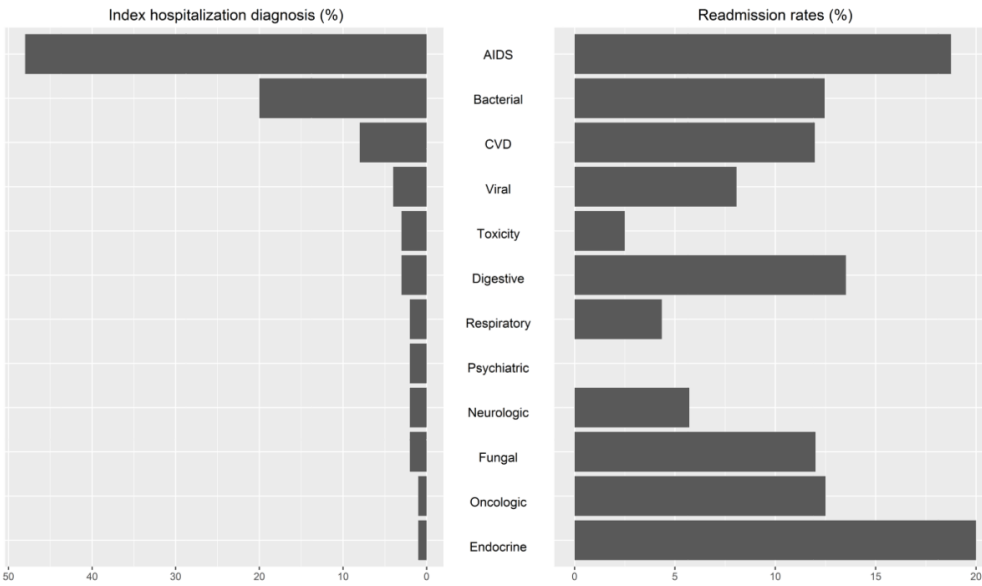

Figure 2. Index hospitalizations causes and 30-day readmission rates per diagnosis Left panel shows proportion of index hospitalizations by cause of hospitalization. Right panel shows readmissions rate by index hospitalization cause. CVD: cardiovascular diseases. 


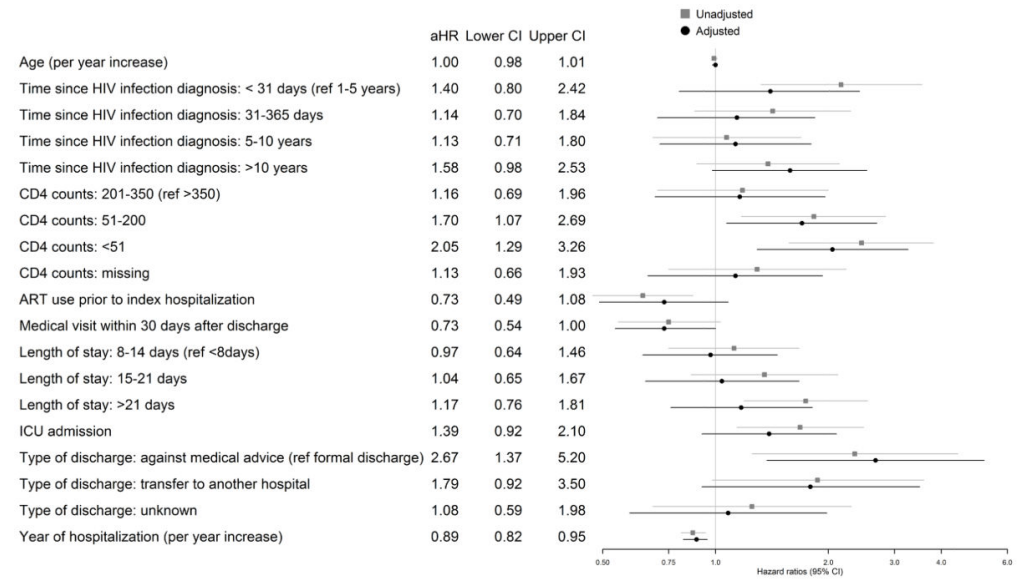

Figure 3. Adjusted extended Cox regression model

Hazard ratios and $95 \%$ confidence intervals for 30-day readmission. 


\section{Table 1}

Socio-demographic and clinical characteristics and unadjusted hazard ratios (95\% confidence interval) for patients who hospitalized stratified by readmission, INI cohort.

\begin{tabular}{|c|c|c|c|c|}
\hline & \multirow{2}{*}{$\begin{array}{l}\text { Index hospitalizations } \\
\text { Total }(\mathrm{N}=1442)\end{array}$} & \multicolumn{2}{|c|}{ 30-day readmission } & \multirow{2}{*}{$\begin{array}{l}\text { Unadjusted models uHR } \\
(95 \% \mathrm{CI})\end{array}$} \\
\hline & & No $\left(N=1240^{a}\right)$ & Yes $(\mathrm{N}=\mathbf{2 0 2})$ & \\
\hline \multicolumn{5}{|l|}{ Sex at birth } \\
\hline Male & $932(64.6)$ & $802(64.7)$ & $130(64.4)$ & ref \\
\hline Female & $510(35.4)$ & $438(35.3)$ & $72(35.6)$ & $1.02(0.75,1.37)$ \\
\hline Age $^{*}$ (years) median (IQR) & $39.9(33.2,47.1)$ & $40(33.3,47.2)$ & $38.8(32.2,46.7)$ & $0.99(.098,1.01)$ \\
\hline$<30$ years & $233(16.2)$ & $195(15.7)$ & $38(18.8)$ & ref \\
\hline 30-39 years & $496(34.4)$ & $428(34.5)$ & $68(33.7)$ & $0.85(0.57,1.27)$ \\
\hline $40-49$ years & $447(31)$ & $388(31.3)$ & $59(29.2)$ & $0.81(0.54,1.22)$ \\
\hline$\supseteq 50$ years & $266(18.4)$ & $229(18.5)$ & $37(18.3)$ & $0.87(0.55,1.36)$ \\
\hline \multicolumn{5}{|l|}{ Race/ethnicity } \\
\hline White & $616(42.7)$ & $536(43.2)$ & $80(39.6)$ & ref \\
\hline Non White & $826(57.3)$ & $704(56.8)$ & $122(60.4)$ & $1.16(0.87,1.56)$ \\
\hline \multicolumn{5}{|l|}{ Educational level } \\
\hline Up to 9 years & $1026(71.2)$ & $878(70.8)$ & $148(73.3)$ & ref \\
\hline More than 9 years & $416(28.8)$ & $362(29.2)$ & $54(26.7)$ & $0.88(0.64,1.22)$ \\
\hline \multicolumn{5}{|l|}{ HIV exposure category } \\
\hline Heterosexual & $900(62.4)$ & $782(63.1)$ & $118(58.4)$ & ref \\
\hline MSM & $446(30.9)$ & $381(30.7)$ & $65(32.2)$ & $1.11(0.82,1.52)$ \\
\hline IDU & $46(3.2)$ & $36(2.9)$ & $10(5)$ & $1.76(0.88,3.51)$ \\
\hline Other/unknown & $50(3.5)$ & $41(3.3)$ & $9(4.5)$ & $1.45(0.71,2.94)$ \\
\hline Time since HIV diagnosis (years) ${ }^{*}$ & $5.4(0.8,11.3)$ & $5.4(0.9,11.3)$ & $4.6(0.3,11.3)$ & \\
\hline$\$ 30$ days & $136(9.4)$ & $106(8.5)$ & $30(14.9)$ & $2.16(1.32,3.55)$ \\
\hline 31-365 days & $257(17.8)$ & $218(17.6)$ & $39(19.3)$ & $1.42(0.88,2.29)$ \\
\hline 1-5 years & $305(21.2)$ & $272(21.9)$ & $33(16.3)$ & ref \\
\hline $5-10$ years & $294(20.4)$ & $260(21)$ & $34(16.8)$ & $1.07(0.68,1.69)$ \\
\hline$>10$ years & $450(31.2)$ & $384(31)$ & $66(32.7)$ & $1.38(0.89,2.14)$ \\
\hline $\mathrm{CD}^{*}\left(\right.$ cells $\left./ \mathrm{mm}^{3}\right)$, median $(\mathrm{IQR})$ & $177(56,395)$ & $191(62,414)$ & $118(32,257)$ & \\
\hline$>\mathbf{3 5 0}$ & $360(25)$ & $327(26.4)$ & $33(16.3)$ & ref \\
\hline 201-350 & $222(15.4)$ & $198(16)$ & $24(11.9)$ & $1.18(0.69,2.01)$ \\
\hline $51-200$ & $391(27.1)$ & $328(26.5)$ & $63(31.2)$ & $1.87(1.20,2.91)$ \\
\hline 50 & $283(19.6)$ & $223(18)$ & $60(29.7)$ & $2.51(1.61,3.92)$ \\
\hline Missing & $186(12.9)$ & $164(13.2)$ & $22(10.9)$ & $1.36(0.80,2.31)$ \\
\hline \multicolumn{5}{|l|}{ HIV viral load ${ }^{*}($ copies $/ \mathrm{mL})$} \\
\hline$\leq 400$ & $499(34.6)$ & $436(35.2)$ & $63(31.2)$ & ref \\
\hline$>400$ & $699(48.5)$ & $597(48.1)$ & $102(50.5)$ & $1.16(0.85,1.60)$ \\
\hline Missing & $244(16.9)$ & 207 (16.7) & $37(18.3)$ & $1.19(0.79,1.79)$ \\
\hline
\end{tabular}




\begin{tabular}{|c|c|c|c|c|}
\hline & \multirow{2}{*}{$\begin{array}{l}\text { Index hospitalizations } \\
\text { Total }(\mathrm{N}=1442)\end{array}$} & \multicolumn{2}{|c|}{ 30-day readmission } & \multirow{2}{*}{$\begin{array}{l}\text { Unadjusted models uHR } \\
(95 \% \text { CI })\end{array}$} \\
\hline & & No $\left(N=1240^{a}\right)$ & Yes $(N=202)$ & \\
\hline No & $362(25.1)$ & $294(23.7)$ & $68(33.7)$ & ref \\
\hline Yes & $1080(74.9)$ & $946(76.3)$ & $134(66.3)$ & $0.64(0.47,0.87)$ \\
\hline Time on ART (years), median (IQR) ${ }^{*} b$ & $4.2(1.1,9.1)$ & $4.2(1.1,9.1)$ & $4.2(0.8,8.6)$ & \\
\hline Length of stay, median (IQR) ${ }^{*}$ & $12(7,22)$ & $12(7,22)$ & $16(8,28)$ & \\
\hline$\unlhd$ days & $12(7,22)$ & $364(29.4)$ & $45(22.3)$ & ref \\
\hline 8-14 days & $409(28.4)$ & $356(28.7)$ & $50(24.8)$ & $1.12(0.75,1.67)$ \\
\hline 15-21 days & $406(28.2)$ & $198(16)$ & $34(16.8)$ & $1.35(0.86,2.12)$ \\
\hline$>21$ days & $232(16.1)$ & $322(26)$ & $73(36.1)$ & $1.74(1.19,2.54)$ \\
\hline \multicolumn{5}{|l|}{ Type of discharge ${ }^{*}$} \\
\hline Medical discharge & $1300(90.2)$ & $1128(91)$ & $172(85.1)$ & ref \\
\hline Against medical advice & $42(2.9)$ & $31(2.5)$ & $11(5.4)$ & $2.35(1.25,4.42)$ \\
\hline Transfer & $33(2.3)$ & $25(2)$ & $8(4)$ & $1.87(0.98,3.59)$ \\
\hline Unknown & $67(4.6)$ & $56(4.5)$ & $11(5.4)$ & $1.25(0.68,2.30)$ \\
\hline ICU admission * & $148(10.3)$ & $117(9.4)$ & $31(15.3)$ & $1.68(1.14,2.48)$ \\
\hline $\begin{array}{l}\text { Medical visit within } 30 \text { days after } \\
\text { discharge }^{c}\end{array}$ & $1002(69.5)$ & $926(74.7)$ & 76 (37.6) & $0.75(0.55,1.03)$ \\
\hline Year of hospitalization & & & & $0.87(0.81,0.94)^{d}$ \\
\hline 2007 & $126(8.7)$ & $101(8.1)$ & $25(12.4)$ & \\
\hline 2008 & $217(15)$ & $177(14.3)$ & $40(19.8)$ & \\
\hline 2009 & $226(15.7)$ & $196(15.8)$ & $30(14.9)$ & \\
\hline 2010 & $211(14.6)$ & $170(13.7)$ & $41(20.3)$ & \\
\hline 2011 & $268(18.6)$ & $241(19.4)$ & $27(13.4)$ & \\
\hline 2012 & $220(15.3)$ & $198(16)$ & $22(10.9)$ & \\
\hline 2013 & $174(12.1)$ & $157(12.7)$ & $17(8.4)$ & \\
\hline
\end{tabular}

MSM: Men who have sex with men; IDU: Injectable drug use; ART: combination antiretroviral therapy; ICU: intensive care unit; uHR: unadjusted hazard ratio.

Refers to index hospitalization or index hospitalization admission date.

Includes 16 patients who died within 30 days (without been readmitted)

$b_{\text {Length of time of ART use prior to index hospitalization admission. }}$

$c$ Defined as a medical visit before end of follow-up or readmission, for those who readmitted.

${ }^{d}$ Year of hospitalization was included in the model as a continuous variable. 